\title{
Effects of Coronary Artery Bypass Grafting Surgery on Olfactory and Taste Functions
}

\author{
Kemalettin Erdem, MD,${ }^{1}$ Erhan Renan Ucaroglu, MD,${ }^{1}$ Abidin Sehitogullari, MD,${ }^{2}$ Ahmet Yuksel, MD,${ }^{1}$ \\ Hikmet Tekce, MD,${ }^{3}$ Yusuf Velioglu, MD,${ }^{1}$ Abdullah Demirhan, MD,${ }^{4}$ Ufuk Turan Kursat Korkmaz, MD ${ }^{1}$ \\ Ferhat Borulu, MD, ${ }^{5}$ Osman Unal, MD,${ }^{1}$ Aynur Ulku, ${ }^{1}$ Ebru Calisir ${ }^{1}$ \\ ${ }^{1}$ Department of Cardiovascular Surgery, Faculty of Medicine, Bolu Abant Izzet Baysal University, Bolu, Turkey; ${ }^{2}$ Departmant of \\ Thoracic Surgery, Faculty of Medicine, Sakarya University, Sakarya, Turkey; ${ }^{3}$ Department of Nephrology, Faculty of Medicine, Bolu \\ Abant Izzet Baysal University, Bolu, Turkey; ${ }^{4}$ Department of Anesthesiology and Reanimation, Faculty of Medicine, Bolu Abant Izzet \\ Baysal University, Bolu, Turkey; ${ }^{5}$ Department of Cardiovascular Surgery, Faculty of Medicine, Ataturk University, Erzurum, Turkey
}

\section{ABSTRACT}

Background: Olfactory and taste sensations have a high impact on the quality of life. Impaired olfactory and taste functions may have a negative effect on physical and mental wellbeing, personal hygiene, and nutritional satisfaction, leading to the occurrence of depressive symptoms and impaired quality of life. Therefore, the recovery period of patients with disturbed olfactory and taste functions may be prolonged, and return to active life may be delayed. We designed this study to determine whether on-pump and off-pump coronary artery bypass grafting (CABG) surgeries have any effects on olfactory and taste functions and compare these functions between the surgical groups.

Methods: A total of 60 patients, who underwent elective isolated first-time CABG, were included in this study. Patients were divided into two groups as Off-Pump and On-Pump CABG groups with 30 patients in each group. In addition to patients' primary clinical and laboratory data, olfactory and taste functions were evaluated pre- and postoperatively in both groups separately, and then these functions were compared between the groups. Olfactory functions were evaluated by the Brief Smell Identification Test, while taste functions by the Burghart Taste test.

Results: Olfactory functions significantly were disrupted in the postoperative period in patients undergoing on-pump CABG $(P$ value $<.05)$, while these functions significantly were not affected in patients undergoing off-pump CABG $(P$ value $>.05)$. During the preoperative period, advanced age and smoking were detected to be independent predictors of impaired olfactory function for the study population. During the postoperative period, smoking, amount of postoperative bleeding and serum low-density lipoprotein (LDL)

Received April 12, 2019; received in revised form August 3, 2019; accepted August 21, 2019.

Correspondence: Kemalettin Erdem, MD, Associate Professor, Department of Cardiovascular Surgery, Faculty of Medicine, Bolu Abant Izzet Baysal University, Golkoy Campus, 14280, Bolu, Turkey; +90-5538981400; fax: +90-374-2534559 (e-mail: drkemalettincvs@yahoo.com). level were found to be independent predictors of impaired olfactory function for just the On-Pump CABG Group. In both groups, no significant deterioration in taste functions occurred during the postoperative period $(P$ value $>.05)$.

Conclusion: Our study demonstrated that olfactory function was impaired in patients, who underwent on-pump CABG in the postoperative period, and significant impairment in taste function was present in neither off-pump nor on-pump CABG patients. However, the results of our study should be supported by more comprehensive, prospective, randomized controlled trials with more extensive patient series and by further tests.

\section{INTRODUCTION}

Cardiovascular diseases are one of the leading causes of death worldwide. Coronary artery bypass grafting (CABG) often is a preferred treatment option for advanced coronary artery diseases. Cardiopulmonary bypass (CPB) frequently is used during CABG. Off-pump CABG without $\mathrm{CPB}$ is another option performed by experienced cardiovascular surgeons as an alternative to this operative strategy [Akarsu 2016; Yuksel 2017; Yuksel 2018].

Many studies have demonstrated that the senses of smell and taste have an essential effect on quality of life and are directly associated with cognitive functions [Frasnelli 2004; Frasnelli 2005; Ciofalo 2015]. In their study, Liu et al [Liu 2016] found taste and smell disorders in $17 \%$ of the general population over the age of 40 years. Also, some studies have reported that taste and smell disorders commonly are seen in patients with cardiovascular disease [Liu 2016; Seubert 2017]. Hence, in our previous study, we demonstrated that olfactory functions tended to deteriorate in the early postoperative period in patients undergoing on-pump CABG with CPB [Erdem 2014].

Patients who underwent off- and on-pump CABG previously have been compared in terms of many adverse events, such as stroke, acute renal dysfunction, atrial fibrillation, and bleeding. However, to the best of our knowledge, there is no study in the literature to compare patients who underwent 
Table 1. The relationship between the operation type with clinical and laboratory parameters of the participants

\begin{tabular}{|c|c|c|c|}
\hline Age (years) & $66.33 \pm 7.68$ & $66.23 \pm 9.06$ & $>.05$ \\
\hline Diabetes mellitus, \% & $16,53.5 \%$ & $11,40.7 \%$ & $>.05$ \\
\hline Smoking, \% & $10,33.3 \%$ & $12,40 \%$ & $>.05$ \\
\hline$\beta$-blocker use, $\%$ & $17,56.6 \%$ & $19,63.3 \%$ & $>.05$ \\
\hline Calcium channel blocker use, $\%$ & $5,16.7 \%$ & $4,13.6 \%$ & $>.05$ \\
\hline Number of vessels & $3.53 \pm 1.16$ & $3.66 \pm 1.09$ & $>.05$ \\
\hline Time to discharge (day) & $5.10 \pm 0.75$ & $4.43 \pm 0.97$ & .04 \\
\hline Postoperative bleeding $(\mathrm{mL})$ & $467.33 \pm 205$ & $260.00 \pm 121$ & $<.01$ \\
\hline Postoperative third day CRP (mg/L) & $128.20 \pm 39$ & $99.83 \pm 20$ & .01 \\
\hline Total cholesterol $(\mathrm{mg} / \mathrm{L})$ & $175.56 \pm 51$ & $197.07 \pm 50$ & $>.05$ \\
\hline Preoperative LDL (mg/L) & $91.18 \pm 22$ & $112.24 \pm 37$ & .01 \\
\hline Antihyperlipidemic therapy, \% & $19,63 \%$ & $13,43 \%$ & $>.05$ \\
\hline Hematocrit (\%) & $38.59 \pm 5$ & $39.05 \pm 4$ & $>.05$ \\
\hline Ejection fraction (\%) & $46.90 \pm 62$ & $48.70 \pm 65$ & $>.05$ \\
\hline
\end{tabular}

ACE: Angiotensin-converting enzyme inhibitor; ARB: Angiotensin receptor blockers; CABG: Coronary artery bypass grafting; CRP: C-reactive protein; LDL: Low density lipoprotein. Values are expressed as mean \pm standard deviation or $\mathrm{N}(\%)$.

off-pump and on-pump CABG operations in terms of the senses of smell and taste. In this study, we aimed to investigate whether olfactory and taste functions were affected in patients, who underwent off-pump and on-pump CABG.

\section{MATERIALS AND METHODS}

Study design and patients: The institutional scientific research ethics committee approved the study protocol. The study was conducted following the principles of the Declaration of Helsinki. All participants were informed of the study, and their written consent was obtained. A total of 60 patients, who underwent elective isolated first-time CABG in our hospital between June 2018 and December 2018 were included in this prospective study. Patients were divided into two groups: Off-Pump and On-Pump CABG groups, with 30 patients in each group. Patients were evaluated pre- and postoperatively for baseline clinical characteristics and laboratory data as well as taste and smell functions; these functions were then compared between the groups. Olfactory functions were evaluated by the Brief Smell Identification Test (B-SIT), and taste functions were evaluated by the Burghart Taste test (Messtechnik GmbH Tinsdaler Weg 17522880 Wedel, Germany). Excluded from the study were patients with a neurodegenerative or psychiatric disorder known to affect smell and taste functions, those with a history of cerebrovascular event, patients with a low intellectual consciousness, those operated previously due to structural nasal disorder or other reasons such as polyp etc., patients with a nasal allergic or infectious diseases, those with renal or hepatic diseases, patients with delayed postoperative extubation ( $>8$ hours), and those who did not want to participate.

An experienced otolaryngologist evaluated smell capacity: Oral and nasal examinations of the patients in the preoperative period. The baseline smell and taste capacities of the patients were recorded on the day before the operation. Smell and taste functions were evaluated and recorded on postoperative 1st and 3rd days. Evaluations included routinely studied clinic, blood, and laboratory tests, amount of postoperative bleeding, and data of blood and blood product transfusion.

B-SIT smell test: The test consists of a booklet of 12 leaves. There is one odorant on each leaf (mint, banana, clove, leather, strawberry, pine, cinnamon, soot, lemon, soap, baby powder, and rose). The smells on the leaves are released when scratched with a pencil tip. Patients were made to smell the odorants from a $2 \mathrm{~cm}$ distance. The odorants were smelled with 1-minute intervals to avoid desensitization. The patients were given 4 alternative options for each odorant and asked to give one correct answer. The participants 
Table 2. The relationship between the operation types and the smell and taste functions of the participants.

\begin{tabular}{|c|c|c|c|}
\hline Total correct odor number preoperatively & $7.43 \pm 1.65$ & $7.30 \pm 1.70$ & .947 \\
\hline Total correct odor number on postoperative third day & $5.86 \pm 1.38$ & $7.40 \pm 1.42$ & $<.01$ \\
\hline Total correct tastes number preoperatively & $4,100 \%$ & $4,100 \%$ & $>.05$ \\
\hline Total correct tastes number on postoperative third day & $4,100 \%$ & $4,100 \%$ & $>.05$ \\
\hline
\end{tabular}

CABG: Coronary artery bypass grafting. Values are expressed as mean \pm standard deviation or $\mathrm{N}(\%)$.

were asked to give 1 correct answer; however, if they were undecided among the four options.

Evaluation of taste capacity: Burghart Taste test was used to evaluate the taste capacity of the entire mouth. The test solutions are found in $25 \mathrm{ml}$ spray bottles. This test included 4 primary flavors (sweet, salty, sour, and bitter). The test was used by spraying once from a distance of $2-3 \mathrm{~cm}$ to reach the entire inside of the mouth. One-minute pauses were taken between tests to prevent desensitization. Participants were asked to give 1 correct answer anyway if they were undecided among the four options.

Surgical technique: All operations were performed with median sternotomy under general anesthesia. Left internal thoracic artery and great saphenous veins routinely were used as bypass grafts. Patients who underwent on-pump CABG were heparinized with 300-400 U/kg heparin sodium. Two-stage right atrial cannulation was carried out through the ascending aorta for CABG. CPB was initiated when activated clotting time $(\mathrm{ACT})>400$ seconds. Cardiac arrest was achieved with initial antegrade and subsequent retrograde blood cardioplegia every 15 minutes. The operations were performed with a single cross-clamp technique at $32-34^{\circ} \mathrm{C}$. The roller pump and membrane oxygenator (Dideco Avant 903) were used for CPB. Heparin sodium was neutralized as $100 \mathrm{U} / 1 \mathrm{mg}$ protamine after CPB. Patients who underwent off-pump CABG were heparinized with $200 \mathrm{U} / \mathrm{kg}$ heparin sodium. The sling sutures were put in both margins of the pericardium and between the left pulmonary veins (posterior pericardium). Distal anastomoses were initiated when ACT $>300$ seconds. Patients were given a trendelenburg position, and the table was turned toward the surgeon during distal anastomoses. Left anterior descending artery, right coronary artery and its branches, circumflex artery and its branches were anastomosed, respectively. Temporary bulldog clamp was applied in the proximal part of the arteriotomy. After the heart returned to its normal anatomic position, proximal anastomoses were made to the ascending aorta using tangential clamps. Heparin sodium was antagonized with 100 $\mathrm{U} / 1 \mathrm{mg}$ protamine. The patients were closed using the same surgical method.

Biochemical evaluation: Patient blood samples were collected the morning of the preoperative and postoperative first and third days. Serum glucose, creatinine, total cholesterol, high-density lipoprotein (HDL), and low-density lipoprotein (LDL) values were evaluated with standard methods in all patients. Blood samples were collected in the antecubital area with an automatic blood collector. Samples were assayed in an automatic analyzer (Beckman Coulter LH 750, Fullerton, CA) within 30 minutes. High-sensitivity C-reactive protein (CRP) levels were evaluated with Architect CI8000 systems (Abbott Laboratories, Abbott Park, IL) using Siemens Prospec analyzer (Siemens Healthcare Diagnostics, Tarrytown, NY).

Statistical analysis: Analyses were performed using Statistical Package for Social Sciences (SPSS Inc., Chicago, Illinois, USA) version 20.0. Normal distribution of the variables was examined with visual (histogram and probability charts) and analytic methods (Kolmogorov-Smirnov / Shapiro-Wilk tests). Descriptive statistics are expressed as mean and standard deviation for normally distributed variables. Descriptive analysis of non-normally distributed variables were carried out using median and interquartile range (IQR). The student $\mathrm{t}$-test was used to determine the relationship between normally distributed parameters. Mann Whitney U test was used for the analysis of non-normally distributed parameters. Correlation coefficients and statistical significances were calculated with the Spearman test for the correlations between olfactory functions, with at least one of them non-normally distributed or ordinal, and clinical and laboratory variables. Effects of different preoperative, postoperative first day and postoperative third-day predictors on olfactory functions were examined using multivariate linear regression analysis. $P<.05$ values were considered statistically significant.

\section{RESULTS}

When patient demographics and clinical features were examined between the On-Pump and Off-Pump groups; time to discharge, postoperative (PO) bleeding amount, amount of blood and blood products transfused, third postoperative day CRP and preoperative LDL values statistically were significantly higher in the On-Pump Group compared with the OffPump Group (Table 1). No statistically significant difference was found between the groups, in terms of other parameters 
Table 3. Bivariate correlation analysis between the odor, taste functions of the cases and the clinical, laboratory parameters

\begin{tabular}{|c|c|c|c|c|c|c|}
\hline Sex & .982 & +0.95 & .91 & 0.01 & .38 & 0.01 \\
\hline Diabetes mellitus & .006 & -0.24 & .05 & 0.36 & .02 & 0.30 \\
\hline ACE or ARB use & .490 & -0.09 & .955 & 0.008 & .706 & -0.05 \\
\hline -blocker use & .898 & 0.017 & .117 & 0.205 & .994 & -0.01 \\
\hline CCB use & .273 & 0.144 & .653 & 0.055 & .768 & -0.039 \\
\hline Number of vessels & .350 & 0.123 & .797 & 0.036 & .546 & 0.080 \\
\hline Discharge & .872 & 0.021 & 0.247 & -0.152 & .162 & -0.183 \\
\hline Postoperative first day CRP & .520 & -0.088 & .513 & -0.086 & .324 & -0.129 \\
\hline Postoperative third day CRP & .200 & 0.168 & .993 & 0.001 & .429 & -0.104 \\
\hline Total cholesterol & .348 & -0.127 & .783 & -0.037 & .802 & 0.2019 \\
\hline Preoperative LDL & .038 & 0.289 & .014 & -0.339 & .007 & -0.371 \\
\hline Antihyperlipidemic therapy & .806 & 0.032 & .654 & 0.059 & .197 & 0.169 \\
\hline Hematocrit & .962 & 0.060 & .300 & 0.136 & .619 & -0.066 \\
\hline Ejection fraction & .119 & 0.145 & .783 & 0.080 & .184 & 0.218 \\
\hline
\end{tabular}

ACE: Angiotensin-converting enzyme inhibitor; ARB: Angiotensin receptor blockers; CABG: Coronary artery bypass grafting; CCB: Calcium channel blocker; CRP: C-reactive protein; LDL: Low density lipoprotein.

(age, gender, smoking, rate of diabetes, drugs used, and biochemical tests, etc.) except for the values mentioned above.

When patient smell and taste functions were compared between the On-Pump and Off-Pump groups, no statistically significant difference was found between the groups, in terms of the total preoperative number of correct odorants $(7.43 \pm$ 1.65 versus $7.30 \pm 1.70, P=.947)$. Postoperative first-day total number of correct odorants $(6.13 \pm 1.45$ versus $7.20 \pm 1.58$, $P=.009)$ and postoperative third-day total number of correct odorants $(5.86 \pm 1.38$ versus $7.40 \pm 1.42, P<.01)$ significantly were higher in the Off-Pump Group (Table 2).

When the total number of correct tastes was examined on every patient's preoperative, postoperative first and postoperative third days, all participants correctly answered all taste tests, and the results were not statistically significant (Table 3 ).

When bivariate correlations between olfactory functions and clinical and laboratory parameters of the patients were examined, a negative correlation was found between preoperative olfactory values and age $(P=.001, \mathrm{R}=-0.54)$, smoking $(P=.001, \mathrm{R}=-0.75), \mathrm{CRP}(P=.01, \mathrm{R}=-0.594)$, and LDL $(P=.038, \mathrm{R}=-0.289)($ Table 3$)$. Postoperative first day olfactory values were negatively correlated with age $(P=.001$, $\mathrm{R}=-0.40)$, smoking $(P=.001, \mathrm{R}=-0.59)$, preoperative CRP $(P=.001, \mathrm{R}=-0.501)$, preoperative LDL $(P=.014, \mathrm{R}=-0.339)$, amount of postoperative bleeding $(P=.001, \mathrm{R}=-0.444)$, and amount of blood and blood products used in the postoperative period $(P=.005, \mathrm{R}=-0.357)$ (Table 2$)$. Again, postoperative third day olfactory values were negatively correlated with the presence of diabetes $(P=.02, \mathrm{R}=-0.30)$, smoking $(P=.001, \mathrm{R}=-0.41)$, amount of postoperative bleeding $(P=.001, \mathrm{R}=-0.523)$, amount of blood and blood products transfused $(P=.001, \mathrm{R}=-0.436)$, and preoperative $\mathrm{LDL}$ $(P=.007, \mathrm{R}=-0.371)$ (Table 2$)$.

The factors affecting olfactory functions on preoperative, postoperative first, and third postoperative days were examined with multivariate regression analysis. Age, smoking, presence of diabetes, amount of postoperative bleeding, CRP, and LDL values that were thought to be associated with smell function were included in the multivariate model created. As the result of these analyses, age ( $P=.047,95 \%$ CI: $-0,089$ to $-0,001)$, LDL $(P=.074,95 \%$ CI: -0.089 to -0.001$)$, and smoking $(P=.001,95 \% \mathrm{CI}:-2.303$ to -1.278$)$ were found to affect preoperative olfactory functions as independent predictors. Smoking $(P=.001,95 \%$ CI: -2.303 to -0.793$)$, LDL $(P=.068$, $95 \%$ CI: $-0,001$ to 0.021$)$, and amount of postoperative bleeding $(P=.016,95 \% \mathrm{CI}$ : -0.005 to -0.001$)$ were found to affect postoperative first day olfactory functions as independent predictors. Smoking ( $P=.045,95 \%$ CI: -1.549 to -0.018$)$, 
Table 4. Multivariate analysis of parameters acting on odor functions

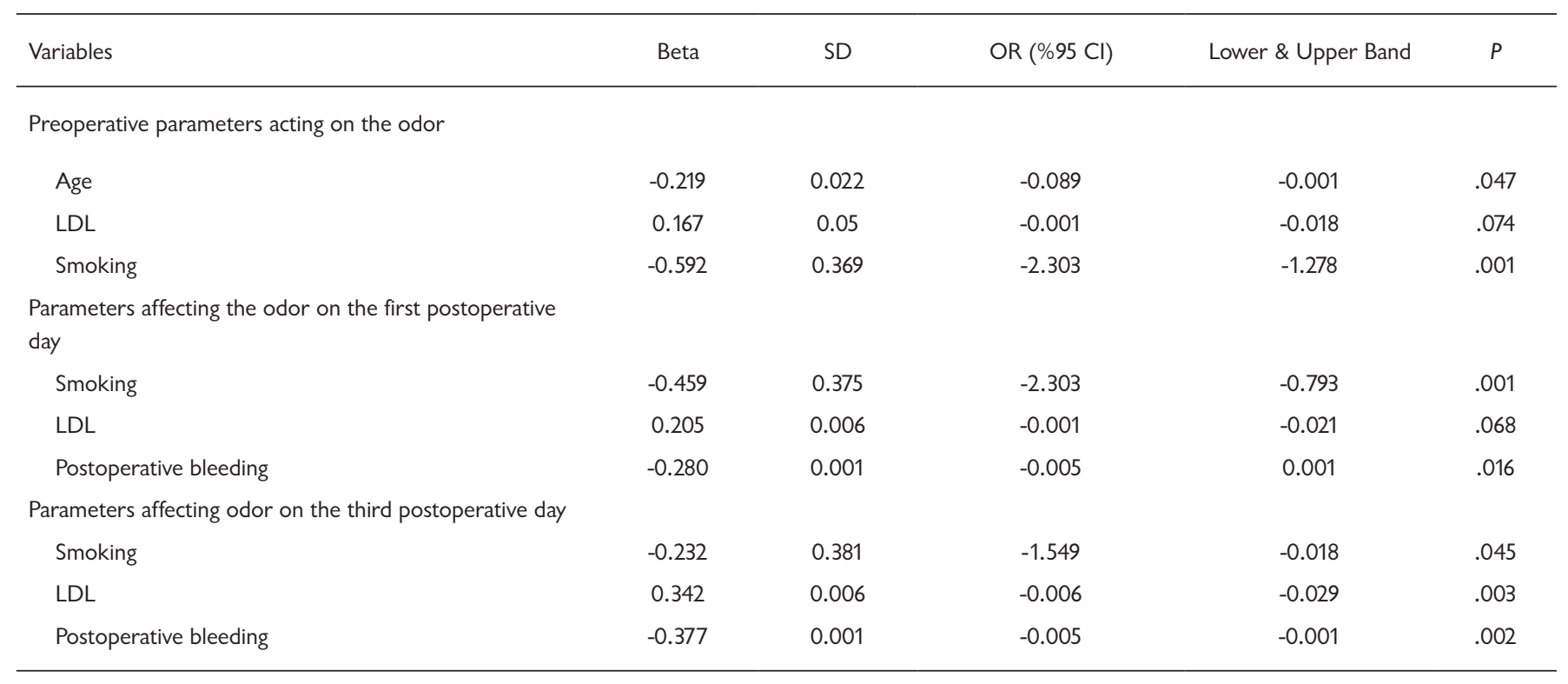

Cl: Confidence interval; LDL: Low density lipoprotein; OR: Odds ratio; SD: Standard deviation

LDL $(P=.003,95 \% \mathrm{CI}: 0.006$ to 0.029$)$, and amount of postoperative bleeding $(P=.002,95 \% \mathrm{CI}$ : -0.005 to -0.001$)$ were found to affect postoperative third day olfactory functions as independent predictors (Table 4).

\section{DIsCUSSION}

Here is a summary of the results from this study:

1. Olfactory functions significantly were affected in the postoperative period in patients who had undergone onpump CABG, although these functions were not significantly influenced in patients who had undergone off-pump CABG.

2. The common factors affecting olfactory functions were determined as the presence of diabetes mellitus, smoking, amount of postoperative bleeding, and LDL levels.

3. While age and smoking were found to be independent predictors for olfactory levels of the patient in the preoperative period, smoking, amount of postoperative bleeding and LDL levels were found to be independent predictors for olfactory levels in the postoperative period.

Olfactory functions are known to directly associate with daily routine activity, cognitive functions, and quality of life. Deterioration of olfactory functions may deteriorate patients' basic functions and activities [Frasnelli 2004; Frasnelli 2005; Ciofalo 2015]. We revealed in our study that deteriorated olfactory functions in on-pump CABG patients may adversely affect the healing process of these patients. In our opinion, it is vital to detect the possible deterioration of olfactory functions early on and to take necessary measures before the deterioration of the basic activities and functions of these patients.

To the best of our knowledge, patients who had undergone on-pump and off-pump CABG were compared for many parameters, but they have never been explicitly compared for smell and taste functions. Also, several studies have investigated the relationship of cognitive functions, according to surgery type, and have shown that cognitive functions were decreased by $5-30 \%$ after on-pump surgery type [Newman 2001; Sun 2012]. The authors explained this directly by inflammation as well as microembolization and changes in perfusion flow [Newman 1996; Moody 1990]. In another study by Sultan et al [Sultan 2011], the authors reported that inflammation is essential in showing adverse effects on olfactory functions. In that experimental study, the effect of tumor necrosis factor $\alpha$, which is among acute phase reactants was investigated in a transgenic mice model, and high tumor necrosis factor- $\alpha$ levels are associated with worsened olfactory functions. Therefore, led by the previous preliminary studies, we investigated the effects of CRP levels, that widely is used in clinical practice, on olfactory levels. As is known, CRP is one of the leading positive acute phase reactants, and it routinely is used in our clinic with this feature. In our previous study, we demonstrated that olfactory functions were deteriorated with CRP levels that were elevated due to inflammation, in patients who had undergone on-pump CABG [Erdem 2014]. In the present study, we confirmed that preoperative CRP values that reflect increased inflammation negatively affected olfactory functions both in the On-Pump and Off-Pump patient groups. Statistical relationship of CRP levels and olfactory functions was in the limit on the third postoperative day, and the limited number of our patients may explain this. Deteriorated olfactory functions in patients undergoing on-pump CABG might be the result of increases in systemic inflammation, microembolism, or change in perfusion pressure, which might be caused by $\mathrm{CPB}$. 
Studies in the literature have reported controversial results about smell and taste functions in a diabetic patient group. In a study by Khobragade et al [Khobragade 2012] comparing 70 patients diagnosed with type 2 diabetes mellitus (DM) with patients in the general population, deterioration of taste function was reported to be higher in patients with type $2 \mathrm{DM}$. In that study, the authors used 4 basic flavor tests, consisting of 7 different half diluted concentrations (The taste threshold was evaluated using 7 different serially half diluted concentrations of glucose $(2.00 \mathrm{M}-0.031 \mathrm{M}), \mathrm{NaCl}(1.00 \mathrm{M}-0.0156 \mathrm{M})$, citric acid $(0.05 \mathrm{M}-0.0007 \mathrm{M})$, and quinine sulphate $(0.001$ $\mathrm{M}-0.000015 \mathrm{M})$. In a study by Rasmussen et al [Rasmussen 2018] comparing DM patients with the general population, it was found that olfactory functions were decreased more in DM patients, this reduced daily food intake, while no significant difference was found in taste functions. In our study, we demonstrated that olfactory function scores were lower in type $2 \mathrm{DM}$ patients compared with patients without type 2 $\mathrm{DM}$, and olfactory functions were deteriorated in the postoperative period. In a prospective controlled study by Dahlslett et al [Dahlslett 2012], they used a 4 basic flavors (sweet, sour, bitter, and salty) test (Burghart, Wedel, Germany) with 4 different concentrations to evaluate taste functions. Gondivkar et al [Gondivkar 2009] used 5 different concentrations of a 4 basic flavors test $(0.01$ to $1.00 \mathrm{~mol} / \mathrm{L})$ and showed that taste function was decreased in type $2 \mathrm{DM}$ patients. In our study, we used a single concentration $(1.00 \mathrm{~mol} / \mathrm{L})$ Burghart taste test (Messtechnik GmbH Weg 17522880 Wedel, Germany) to evaluate taste functions. In our study, the taste concentration we used was high.

Although the negative effect of smoking on taste and smell function has long been known, there are controversial results in the literature on this issue. In a recent study by Rasmussen et al [Rasmussen 2018], it was shown that smoking lead among the factors to negatively affect olfactory functions in adults with diabetes. In that large study, besides smoking, age, BMI, and alcohol consumption were found as independent predictors for olfactory dysfunction. On the other hand, Huang et al [Huang 2017] could not show a similar correlation. Whereas olfactory functions have been shown in various patient populations, the functions have not been studied in patients undergoing CABG operation. In our study in all statistical analyses, we showed the correlation between smoking and deteriorated olfactory functions in this patient population. Furthermore, this negative correlation was obtained both for bivariate correlation analyses and multivariate analyses. Smoking is the most important predictor for negative olfactory functions in CABG patients. Moreover, this negative effect of smoking with elevated LDL includes both the preoperative and postoperative periods, whereas effects to other parameters are limited to preor postoperative olfactory functions.

Many studies in the literature have shown that the amount of postoperative bleeding is higher in on-pump surgery compared with off-pump surgery, and thus more blood products are used with this technique [van Dijk 2001; Hussain 2016; Wang 2017]. Consistently with the literature, in our study, higher amounts of postoperative bleeding and the use of blood products were observed in the On-Pump Group. Besides the higher amount of postoperative bleeding and the use of blood products, the $\mathrm{CPB}$ system itself is known to be associated with increased inflammatory response [Suleiman 2008; Kim 2003; Kawamura 1993]. In our study, a negative correlation between bleeding and blood transfusions and postoperative olfactory functions was detected. Consequently, the relationship between the decrease in olfactory functions and the increase in inflammation might be explained by this negative correlation between increased postoperative bleeding and deteriorated olfactory functions. On the other hand, although postoperative values of CRP, which is a marker of systemic inflammatory response were not determined as an independent predictor in multivariate analysis, CRP levels significantly were higher on the third postoperative day.

It has been reported in literature that smell and taste functions deteriorate with blood lipid level and age in the general population, and at the same time, people with smell and taste dysfunctions have higher blood lipid and lipoprotein concentrations [Cai 2014; Lee 2013]. Huang et al. [Huang 2017] showed that deterioration in olfactory functions significantly was associated with high serum total cholesterol level, age, and smoking. In our study, we found that high total cholesterol levels showed no negative effect on olfactory functions, but high serum levels of LDL, which plays a more critical role in atherosclerosis and is a subparameter of total cholesterol, showed a negative effect on olfactory functions. Results of our study demonstrate a clinical reflection of molecular studies showing that lipid metabolism and smell and taste functions are regulated by the same hormones and neuropeptides [Martin 2009; Kawai 2000; Shin 2008].

Our study has several limitations. First, the number of patients was relatively small. Second, we could not precisely demonstrate changes in taste functions because of the differences in the methodology we used in taste tests. Third, we could not investigate the effect of intubation and extubation durations of olfactory functions. Finally, we did not take into account the cognitive functions and sociocultural levels of the patients.

In conclusion, we suggest that more bleeding occurred in the postoperative period, causing a higher amount of blood and blood products transfused and the effect of $\mathrm{CPB}$ olfactory functions were more deteriorated in patients who had undergone on-pump CABG. Also, we found that smoking and serum LDL levels that play a role in the development of atherosclerosis and diabetes mellitus were independent predictors on decreased olfactory functions. We found that onpump CABG did not cause a decrease in taste functions. We believe that these results should be supported by further comprehensive studies with a more extensive series of patients and more advanced laboratory investigations.

\section{REFERENCES}

Akarsu S, Kan II, Sevingil T, Tok M. 2016. Effect of body mass index on mortality and morbidity in patients undergoing coronary artery bypass grafting surgery. Heart Surg Forum 19:218-23. 
Cai H, Maudsley S, Martin B. 2014. What is the role of metabolic hormones in taste buds of the tongue. Front Horm Res 42:134-46.

Ciofalo A, Zambetti G, Romeo M, et al. 2015. Taste and olfaction in middle ear surgery. Ann Otol Rhinol Laryngol 124:312-6.

Dahlslett SB, Goektas O, Schmidt F, et al. 2012. Psychophysiological and electrophysiological testing of olfactory and gustatory function in patients with multiple sclerosis. Eur Arch Otorhinolaryngol 269:1163-9.

Erdem K, Yurttas V, Bilgi M, et al. 2014. Evaluation of olfactory memory after coronary artery bypass grafting. Kardiochir Torakochirurgia Pol 11:381-4.

Frasnelli J, Hummel T. 2005. Olfactory dysfunction and daily life. Eur Arch Otorhinolaryngol 262:231-5.

Frasnelli J, Landis BN, Heilmann S, et al. 2004. Clinical presentation of qualitative olfactory dysfunction. Eur Arch Otorhinolaryngol 261:411-5.

Gondivkar SM1, Indurkar A, Degwekar S, Bhowate R. 2009. Evaluation of gustatory function in patients with diabetes mellitus type 2. Oral Surg Oral Med Oral Pathol Oral Radiol Endod 108:876-80.

Huang Z, Huang S, Cong H, et al. 2017. Smell and taste dysfunction is associated with higher serum total cholesterol concentrations in Chinese adults. J Nutr 147:1546-51.

Hussain G, Azam H, Baig MA, Ahmad N. 2016. Early outcomes of onpump versus off-pump coronary artery bypass grafting. Pak J Med Sci 32:917-21.

Kawai K, Sugimoto K, Nakashima K, Miura H, Ninomiya Y. 2000. Leptin as a modulator of sweet taste sensitivities in mice. Proc Natl Acad Sci U S A 97:11044-9.

Kawamura T, Wakusawa R, Okada K, Inada S. 1993. Elevation of cytokines during open heart surgery with cardiopulmonary bypass: participation of interleukin 8 and 6 in reperfusion injury. Can J Anaesth 40:1016-21.

Khobragade RS, Wakode SL, Kale AH. 2012. Physiological taste threshold in type 1 diabetes mellitus. Indian J Physiol Pharmacol 56:42-7.

Kim WY, Hura M, Chob MS, Leec HS. 2003. Effect of olfactory function on nutritional status of Korean elderly women. Nutr Res 23:723434.

Lee WH, Wee JH, Kim DK, et al. 2013. Prevalence of subjective olfactory dysfunction and its risk factors: Korean National Health and Nutrition Examination Survey. PLoS One 8:e62725.

Liu G, Zong G, Doty RL, Sun Q. 2016. Prevalence and risk factors of taste and smell impairment in a nationwide representative sample of the US population: a cross-sectional study. BMJ Open 6:e013246.
Martin B, Maudsley S, White CM, Egan JM. 2009. Hormones in the nasooropharynx: endocrine modulation of taste and smell. Trends Endocrinol Metab 20:163-70.

Moody DM, Bell MA, Challa VR, Johnston WE, Prough DS. 1990. Brain microemboli during cardiac surgery or aortography. Ann Neurol 28:477-96.

Newman MF, Wolman R, Kanchuger M, et al. 1996. Multicenter preoperative stroke risk index for patients undergoing coronary artery bypass graft surgery. Circulation 94:74-80.

Newman MF, Kirchner JL, Phillips-Bute B, et al. 2001. Longitudinal assessment of neurocognitive function after coronary-artery bypass surgery. N Engl J Med 344: 395-402.

Rasmussen VF, Vestergaard ET, Hejlesen O, Andersson CUN, Cichosz SL. 2018. Prevalence of taste and smell impairment in adults with diabetes: A cross-sectional analysis of data from the National Health and Nutrition Examination Survey (NHANES). Prim Care Diabetes 12:453-9.

Seubert J, Laukka EJ, Rizzuto D, et al. 2017. Prevalence and correlates of olfactory dysfunction in old age: a population-based study. J Gerontol A Biol Sci Med Sci 7:a018994.

Shin Y, Martin B, Golden E, et al. 2008. Modulation of taste sensitivity by GLP-1 signaling. J Neurochem 106:455-63.

Suleiman MS, Zacharowski K, Angelini GD. 2008. Inflammatory response and cardioprotection during open-heart surgery: the importance of anaesthetics. Br J Pharmacol 153:21-33.

Sultan B, May LA, Lane AP. 2011. The role of TNF- in inflammatory olfactory loss. Laryngoscope 121:2481-6.

Sun JH, Wu XY, Wang WJ, Jin LL. 2012. Cognitive dysfunction after off-pump versus on-pump coronary artery bypass surgery: a meta-analysis. J Int Med Res 40:852-8.

van Dijk D, Nierich AP, Jansen EW, et al. 2001. Early outcome after offpump versus on-pump coronary bypass surgery: results from a randomized study. Circulation 104:1761-6.

Wang Y, Shi X, Du R, Chen Y, Zhang Q. 2017. Off-pump versus onpump coronary artery bypass grafting in patients with diabetes: a metaanalysis. Acta Diabetol 54:283-92.

Yuksel A, Kan II, Yolgosteren A, et al. 2017. Are the early postoperative outcomes of coronary artery bypass grafting surgery in elderly women worse compared to men's? Braz J Cardiovasc Surg 32:191-6.

Yuksel A, Yolgosteren A, Kan II, et al. 2018. A comparison of early clinical outcomes of off-pump and on-pump coronary artery bypass grafting surgery in elderly patients. Acta Chir Belg 118:99-104. 